\title{
Impact of cognitive therapy on internalised stigma in people with at-risk mental states
}

\author{
Anthony P. Morrison, Max Birchwood, Melissa Pyle, Clare Flach, Suzanne L. K. Stewart, \\ Rory Byrne, Paul Patterson, Peter B. Jones, David Fowler, Andrew I. Gumley and Paul French
}

\section{Background}

Internalised stigma in young people meeting criteria for at-risk mental states (ARMS) has been highlighted as an important issue, and it has been suggested that provision of cognitive therapy may increase such stigma.

\section{Aims}

To investigate the effects of cognitive therapy on internalised stigma using a secondary analysis of data from the EDIE-2 trial.

\section{Method}

Participants meeting criteria for ARMS were recruited as part of a multisite randomised controlled trial of cognitive therapy for prevention and amelioration of psychosis. Participants were assessed at baseline and at 6, 12, 18 and 24 months using measures of psychotic experiences, symptoms and internalised stigma.

\section{Results}

Negative appraisals of experiences were significantly reduced in the group assigned to cognitive therapy (estimated difference at 12 months was $-1.36(95 \% \mathrm{Cl}$ -2.69 to -0.02$), P=0.047)$. There was no difference in social acceptability of experiences (estimated difference at 12 months was $0.46,95 \% \mathrm{Cl}-0.05$ to $0.98, P=0.079$ ).

\section{Conclusions}

These findings suggest that, rather than increasing internalised stigma, cognitive therapy decreases negative appraisals of unusual experiences in young people at risk of psychosis; as such, it is a non-stigmatising intervention for this population.

\section{Declaration of interest}

None.
Reliable and valid criteria are available to identify help-seeking individuals who are at increased risk of developing schizophrenia and related psychoses. Yung and colleagues ${ }^{1}$ developed such criteria to identify three subgroups experiencing an at-risk mental state (ARMS) for psychosis in individuals aged 14-35 years: two subgroups with state risk factors, defined by the presence of either transient psychotic symptoms or attenuated (subclinical) psychotic symptoms; the other subgroup with trait-plus-state risk factors. These criteria have now been widely adopted internationally, and there have been recent debates about the utility and potential stigma ${ }^{2}$ of including an attenuated psychosis syndrome in DSM-5. ${ }^{3,4}$ Several randomised controlled trials (RCTs) have evaluated the impact of psychosocial and pharmacological interventions ${ }^{5-12}$ on transition to psychosis and severity of psychotic symptoms within this population, and a recent metaanalysis concluded that cognitive therapy may offer a particularly encouraging approach to prevention of psychosis. ${ }^{13}$ However, there is debate about the ethics and unintended consequences of identifying people as being at risk of psychosis and providing any treatment to such a population. ${ }^{14}$ Whereas there are clear adverse effects associated with pharmacological interventions such as antipsychotics, ${ }^{15}$ the risk of iatrogenic harm may be lower with psychosocial interventions. The Early Detection and Intervention Evaluation 2 (EDIE-2) trial recently demonstrated that cognitive therapy seems to be an acceptable treatment, with a high adherence rate. It found that although cognitive therapy did not significantly reduce transition to psychosis or symptom-related distress (in the context of a low overall transition rate), it did reduce severity of psychotic symptoms in people at high risk. ${ }^{12}$ Another notable finding was that the majority of participants in both groups improved over time. However, it has been suggested that psychological treatments such as cognitive therapy may unintentionally increase internalised stigma within this population. ${ }^{2,14}$ The opposite has also been argued in relation to cognitive therapy, with claims that the use of a normalising approach to understanding psychotic experiences should reduce internalised stigma. ${ }^{16,17}$ Internalised stigma has been defined as 'becoming aware of the label and identifying with the stereotypes'18 or 'the internalisation of shame, blame, hopelessness, guilt and fear of discrimination associated with mental illness, ${ }^{19}$ and encompasses the idea that those with mental health problems experience both shame of their diagnosis and fear of discrimination..$^{20}$ Given that this area is unexplored in relation to people identified as being at risk of psychosis, our current study uses a secondary analysis of data from the EDIE- 2 trial ${ }^{12}$ in order to test the hypothesis that in comparison to a symptom-monitoring control, cognitive therapy will reduce internalised stigma in young people with an ARMS.

\section{Method}

\section{Trial design}

The EDIE-2 trial is a multisite randomised, controlled, singleblind (rater) study comparing two conditions (cognitive therapy plus mental state monitoring $v$. a mental state monitoring control). The randomisation algorithm used randomised permuted blocks with block sizes of six or eight, after first stratifying by site and gender. Assessors were masked to treatment condition and many diverse strategies were employed to achieve masked ratings. More details of the trial methodology, including a CONSORT flow diagram, are available in the primary outcome report. $^{12}$

\section{Participants}

Entry criteria for the EDIE-2 trial $^{12}$ were assessed using the Comprehensive Assessment of At-Risk Mental States (CAARMS), which is specifically designed for the assessment of ARMS. ${ }^{21}$ The entry routes consist of individuals experiencing brief limited intermittent psychotic symptoms, attenuated psychotic symptoms 
or state-plus-trait factors. Other inclusion criteria were aged 14-35 and help-seeking. Five sites were involved: Manchester, Birmingham/Worcestershire, Glasgow \& Clyde, Cambridgeshire and Norfolk. Participants were predominantly identified by health professionals working within diverse agencies within primary- and secondary-care settings; our most common sources were early intervention services (34\%), primary care (GPs/primary care mental health teams: $21 \%$ ) and youth/student counselling services (15\%). We recruited 288 participants; 144 were allocated to cognitive therapy and 144 to monitoring only.

\section{Measures}

Eligibility for the study was measured using the CAARMS, ${ }^{21}$ which provided measures of transition to psychosis, symptom severity and associated distress. The interrater reliability of the CAARMS assessments was assessed regularly over the lifetime of the trial and intraclass correlation coefficients indicated good interrater reliability (mean 0.90 , s.d. $=0.03$ ).

Internalised stigma was measured using the Personal Beliefs about Experiences Questionnaire (PBEQ; details available from the authors on request), which is a 13-item self-report questionnaire; each item reflects social and cultural beliefs/stereotypes about psychosis (for example 'I can talk to most people about my experiences'). Participants rate the degree to which they endorse statements to be true about themselves on a four-point scale (one to four): 'strongly disagree', 'disagree', 'agree' and 'strongly agree'. The PBEQ is an adapted version of the Personal Beliefs about Illness Questionnaire (PBIQ) $;{ }^{22,23}$ minor revisions were made in order to make the questionnaire suitable for use with people meeting criteria for ARMS. These revisions involved replacing the word 'illness' with 'experience' and the removal of three items that were not applicable to the ARMS population. Exploratory factor analysis of the PBEQ with the ARMS population has suggested two subscales for this measure: negative appraisals of unusual experiences and the perceived social acceptability of unusual experiences. Analyses indicated good reliability for the negative appraisals subscale $(\alpha=0.74)$ and acceptable reliability for the social acceptability subscale $(\alpha=0.52)$, which is comparable to the reliability of the original subscales of the PBIQ. Items loading highly on the negative appraisals factor included: 'There is something strange about me which is responsible for these experiences', 'It is hard for me to work or keep a job because of my experiences' and 'There must always have been something wrong with me as a person (to have caused these experiences)'. Items loading highly on the social acceptability factor included: 'I can talk to most people about my experiences', 'My experiences mean that I should be kept away from others' and 'I am embarrassed to talk about my experiences' (the latter two items being reverse scored). High scores on negative appraisals indicate higher internalised stigma, whereas lower scores on social acceptability indicate higher internalised stigma.

All participants received assessments that included both CAARMS and PBEQ at baseline, 6 months (end of treatment) and 12 months. Our variable follow-up period meant that participants recruited in the first 14 months of the study were planned to receive 24 months of follow-up; participants recruited after that were offered steadily reducing follow-up periods, depending on time of recruitment, with a minimum follow-up period of 12 months.

\section{Procedure}

Participants were randomised to one of two conditions: monitoring control or cognitive therapy plus monitoring. All participants received treatment as usual plus regular monitoring (incorporating a CAARMS assessment from a research assistant), which represents an enhancement over routine care since it aimed to provide warm, empathic and non-judgemental face-to-face contact, supportive listening, signposting to appropriate local services for unmet needs and crisis management when required. In addition to this monitoring component, participants allocated to the therapy arm of the trial received cognitive therapy based on a specific cognitive model. ${ }^{24}$ Sessions were offered on a weekly basis for up to 26 weeks, plus up to four booster sessions in the subsequent 6 months. Cognitive therapy allows an individualised, problem-oriented approach and incorporates a process of assessment and formulation, which is manualised. The specific interventions depend on individual goals and formulations, but the range of permissible interventions is described in our published manual. ${ }^{16}$ Key ingredients of the approach are the development of a problem and goal list, early formulation (both longitudinal and maintenance), a focus on normalising psychotic-like experiences and an active therapy stance utilising behavioural experiments and evaluation of appraisals. The emphasis on provision of normalising information regarding unusual experiences and the evaluation of catastrophic and shame-related or pessimistic appraisals of such experiences is particularly relevant to this study. Further details regarding the trial design and primary trial outcomes can be found elsewhere. $^{12}$

\section{Statistical methods}

Analyses were undertaken in Stata (version 12) for Windows. The analysis strategy of the primary trial outcome was replicated for this investigation of changes in internalised stigma; primary analysis was by intention-to-treat. Repeated measures models with random effects were used with the summed PBEQ subscales as dependent variables, allowing for attrition and the variable follow-up times introduced by the design of the trial. This approach accounts for non-independence of measures within the same person. The use of these models allows for the analysis of all available data, on the assumption that data are missing at random, ${ }^{25}$ conditional on adjustment for centre and observed baseline scores. The models allowed for linear, quadratic and cubic trends in stigma scores over time, but only involved testing the treatment by linear trend interaction (i.e. based on the assumption that quadratic and cubic trends would be the same for both groups). The estimated parameters include a main effect of treatment, a linear and quadratic effect of follow-up time (months) and a linear effect of treatment $\times$ month interaction. Months of follow-up were centred on 12 months so that the main effect of treatment corresponds to the difference between the two arms determined at 12 months. We adjust for the stigma subscale scores at baseline and the site of the participant. We report robust standard errors, significance levels and confidence intervals.

In order to examine the effect of attendance at cognitive therapy, instrumental variable regression using the adjusted treatment-received algorithm was used in a two-stage least squares analysis on the 12-month outcomes. A regression model was first fitted for the effects of site and randomised group on sessions. Predicted values and residuals from this model were saved and used in a second regression model for the effects of sessions on outcome using the predicted values, allowing additionally for site and the baseline value of the stigma score. This two-stage procedure allows for missing outcomes assuming that they are missing at random. ${ }^{26}$ 


\section{Results}

The baseline characteristics of the whole sample, and the two groups, are presented in Table 1. More detailed baseline characteristics, demographics and referral pathways for the participants are described elsewhere. ${ }^{27}$ The participants allocated to cognitive therapy received a mean of 9.11 sessions (s.d. $=6.69$, range 0-26), each session lasting on average $1 \mathrm{~h}$. Adherence to cognitive therapy was reasonably good, with only 9/144 (6.25\%) not attending any sessions, and 108/144 (75\%) receiving at least four or more sessions. Table 2 shows the results of the PBEQ subscales (negative appraisals and social acceptability) at the 6-, 12-, 18- and 24-month end-points.

The random-effects regression analysis estimates that negative appraisal scores are 1.36 points lower in the cognitive therapy group at 12 months compared with the monitoring group (95\% CI -2.69 to $-0.02, P=0.047)$. There is an overall reduction in negative appraisals over time (coefficient, $-0.18,95 \%$ CI -0.32 to $-0.04, P=0.012)$, but no significant interaction by treatment arm (coefficient, $0.05,95 \% \mathrm{CI}-0.07$ to $0.17, P=0.402$ ). Thus, a beneficial effect of cognitive therapy is observable at 12 months, but the scores of both groups reduced significantly over the whole duration of the trial.

There was no statistically significant difference in social acceptability scores between the cognitive therapy and control arms (coefficient, $0.46,95 \% \mathrm{CI}-0.05$ to $0.98, P=0.079$ ). Social acceptability shows a small increase over time, although this is not quite statistically significant (coefficient, $0.06,95 \%$ CI 0.00 to $0.12, P=0.051$ ). There was no interaction between time and the treatment group, indicating that changes in social acceptability over time do not differ between the groups (coefficient, 0.0001, $95 \%$ CI -0.06 to $0.06, P=0.997$ ).

With regard to the effects of number of sessions, no significant exposure effect was observed at the 12-month outcome for either negative appraisals (sessions effect, $-0.06,95 \% \mathrm{CI}-0.26$ to 0.13 , $P=0.521$ ) or social acceptability (sessions effect, $0.06,95 \% \mathrm{CI}$ -0.02 to $0.14, P=0.132$ ). Thus, there was no dose-response effect.

\section{Discussion}

\section{Main findings}

This is the first study to examine the effects of any treatment on internalised stigma in people with ARMS. Our study has shown that cognitive therapy for people meeting ARMS criteria does not increase, but probably reduces, their negative appraisals of unusual experiences over 12-24 months. Cognitive therapy does not significantly improve their appraisals of the social acceptability of these experiences; however, there was a strong trend in that direction $(P=0.07)$, which is clearly incompatible with suggestions that it may worsen concerns about the social acceptability of psychotic experiences. These results suggest that concerns that the provision of cognitive therapy to people at risk of psychosis may be contraindicated or harmful on the basis of increasing internalised stigma ${ }^{14}$ are unfounded. Indeed, it would seem that, rather than increasing stigma as an adverse effect associated with treatment, cognitive therapy tends to reduce internalised stigma as has been suggested elsewhere. ${ }^{16,17}$

This finding has implications for the likely cost-benefit ratios associated with providing specific treatments for this group, which has been identified as of crucial importance in the ethics of intervening with an at-risk population. ${ }^{28}$ It is, therefore, important that future studies evaluating treatments for ARMS should measure internalised stigma to inform the consideration of such risk-benefit ratios, as they are likely to differ significantly between treatment approaches. It has been argued that a key mechanism of action underpinning the effectiveness of cognitive therapy is the reduction of potentially harmful appraisals of psychotic experiences via the development of normalising, decatastrophising and deshaming understanding of these experiences. ${ }^{16}$ Our findings are compatible with such an assumption.

The non-significant increase in perceived social acceptability of experiences may reflect the possibility that changing appraisals of social acceptability is a greater challenge, since such appraisals are likely to be deep-rooted in the wider cultural context, rather than internally generated. In fact, it may be that a certain level of caution about disclosure of psychotic experiences in the current cultural environment may be adaptive, given the extent of prejudice and discrimination. ${ }^{29}$ Goffman originally described stigma as 'an attribute which is deeply discrediting' and as 'an undesired differentness', and described internalised stigma as identification with a negative stereotype. ${ }^{30}$ Psychosis is one of the most stigmatised mental health problems ${ }^{29,31}$ and stigma associated with psychosis can discourage people from seeking help. ${ }^{32}$ This may delay treatment and leads to social isolation and reduced employment and education opportunities. ${ }^{29}$ Such stigma also results in poorer physical healthcare, suicidality and higher mortality rates. ${ }^{33}$ People with psychosis report internalising stigmatising social stereotypes and experience shame and fear as a consequence. ${ }^{34,35}$ There is a strong negative relationship between internalised stigma and a range of psychosocial variables including hope, self-esteem, empowerment and adherence with treatment; ${ }^{36}$ similar associations have recently been demonstrated in the ARMS population. Therefore, a reduction in negative appraisals of unusual experiences and a trend towards increasing the perceived social acceptability of such experiences is an important finding.

\section{Limitations}

Our trial is the largest trial with an ARMS population to date, and the use of five sites should ensure the generalisability of our

\begin{tabular}{|c|c|c|c|}
\hline & $\begin{array}{l}\text { Whole sample } \\
\qquad(n=288)\end{array}$ & $\begin{array}{l}\text { Cognitive therapy plus } \\
\text { monitoring group } \\
\qquad(n=144)\end{array}$ & $\begin{array}{l}\text { Monitoring only group } \\
\qquad(n=144)\end{array}$ \\
\hline Age, years: mean (s.d.) & $20.74(4.34)$ & $20.73(4.18)$ & $20.75(4.50)$ \\
\hline Male : female ratio & 180:108 & $89: 55$ & $91: 53$ \\
\hline \multicolumn{4}{|c|}{ Comprehensive Assessment of At-Risk Mental States, mean (s.d.) } \\
\hline Severity, summed & $43.06(18.87)$ & $43.50(17.65)$ & $42.61(20.07)$ \\
\hline Distress, summed & $42.61(20.03)$ & $42.77(20.51)$ & $42.45(19.62)$ \\
\hline \multicolumn{4}{|c|}{ Personal Beliefs about Experiences Questionnaire, mean (s.d.) } \\
\hline Negative appraisals & $23.95(4.25)$ & $24.46(4.06)$ & $23.41(4.39)$ \\
\hline Social acceptability & $9.22(2.01)$ & $8.85(2.01)$ & 9.59 (1.99) \\
\hline
\end{tabular}




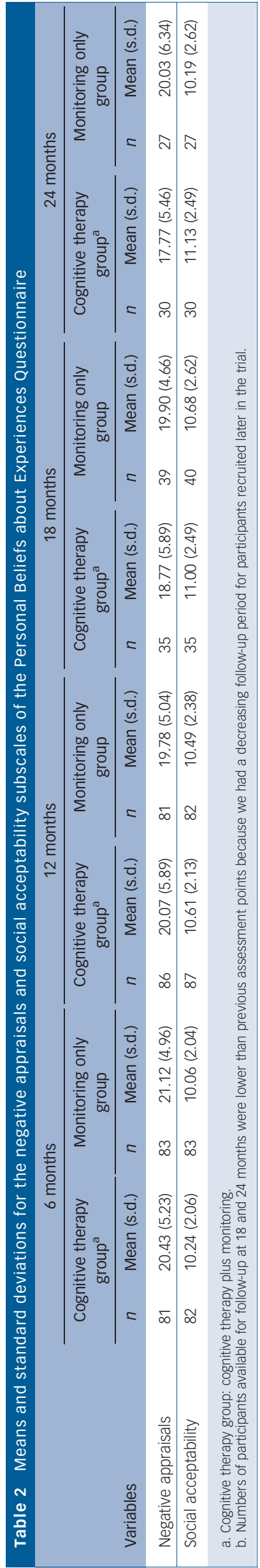

findings to routine clinical service provision. However, there are some potential difficulties with this study. Our measurement of internalised stigma is potentially problematic, since the PBEQ utilised 'my experiences' rather than a label such as 'illness' (as in the original questionnaire), since this seemed more appropriate for an at-risk group yet to establish a diagnosable disorder. However, measuring internalised stigma without reference to a label or diagnosis may be difficult. Nevertheless, participants in the trial were aware that their psychotic-like symptoms were believed to increase their risk of psychosis and cognitive therapy was intended to reduce this and the component symptoms. In this context, we believe the present study provided a strong test of the stigma hypothesis. We should also point out that the social acceptability subscale had only moderate internal reliability, with an alpha of 0.52; however, the original PBIQ subscales had comparable reliability coefficients and it was the negative appraisals subscale that demonstrated a significant beneficial effect of cognitive therapy.

There was a significant proportion of missing data, which can introduce the possibility of bias, however, the proportions were similar for both groups, and much of the missing data were planned due to our variable length follow-up. It would have been interesting to examine the effects of transition to psychosis on internalised stigma but the rarity of this event within our sample prohibits this (only $8 \%$ developed psychosis over the lifetime of the trial). A final consideration is the clinical significance of the demonstrated improvement; it could be argued that an average reduction of 1.36 points on the negative appraisals subscale (roughly corresponding to a change from agree to disagree on one negative stereotype) is not clinically significant. However, our results clearly demonstrate that provision of cognitive therapy does not increase negative appraisals of attenuated psychotic experiences or reduce their perceived social acceptability (i.e. with respect to internalised stigma, cognitive therapy is a benign intervention).

\section{Implications}

We have demonstrated a statistically significant reduction in internalised stigma as a result of cognitive therapy. However, it remains to be seen whether the risks of external stigma and discrimination (such as that experienced from employers, families, health professionals and the general public), which are significant concerns for people with a diagnosis of schizophrenia, ${ }^{30}$ may be associated with a label such as ARMS or the attenuated psychosis syndrome proposed in DSM $-5 .^{3}$ Similarly, as we did not have a group who met criteria but were not informed of this fact, we cannot be sure that the influence of being assessed for risk of psychosis does not, in itself, increase internalised stigma. However, it is worth noting that the levels of stigma in both groups reduced over time (with a significant reduction in negative appraisals of unusual experiences and a strong trend, $P=0.051$, for an increase in perceived social acceptability). This suggests that a non-catastrophic, non-judgemental and empathic approach to engaging such individuals is unlikely to result in increased internalised stigma. This is consistent with data from qualitative interviews of trial participants, ${ }^{37}$ who consistently reported the benefits of normalising, destigmatising messages delivered directly by cognitive therapy and indirectly by regular monitoring of unusual experiences and beliefs. The latter appeared to occur as participants inferred from the regular questions about unusual experiences that many people must experience them and, therefore, they are not as unusual or different as they had originally believed. ${ }^{37}$ Thus, active monitoring within a normalising framework may also be beneficial, and would be benign, easy to 
implement and consistent with International Early Psychosis Association guidelines regarding treatment in the at-risk phase. ${ }^{38}$

There are several other clinical implications of this study. Cognitive therapy reduces negative appraisals of psychotic experiences, which should provide direct benefit given the toxic nature of internalised stigma. Cognitive therapy aims to encourage a normalising, non-catastrophic perspective on the understanding of psychotic experiences within a collaborative framework, which may be the mechanism by which such negative appraisals are reduced. It may be that targeting the social acceptability of such experiences more directly, using methods derived from the literature on prevention of stigma and discrimination (such as contact with other service users, identification of positive role models and celebration of difference), may result in greater effects. However, it is also important to consider that, until wider societal views of psychosis and mental health problems are improved, a cautious approach to disclosure of such experiences may be appropriate. Thus, it is essential that any individual approaches, such as cognitive therapy, are delivered in a context that includes campaigns designed to influence the general public.

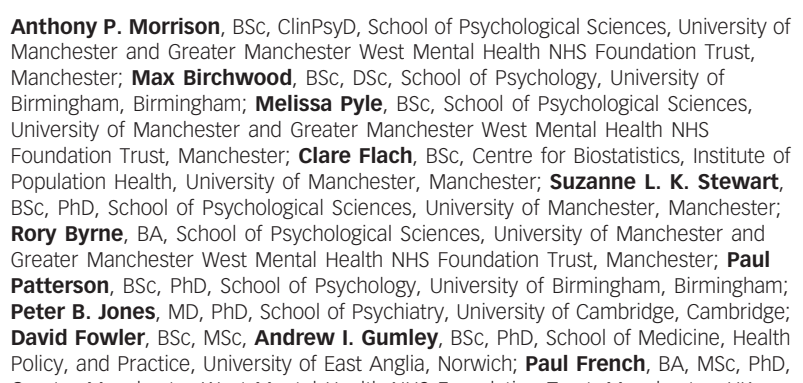

Policy, and Practice, University of East Anglia, Norwich; Paul French, BA, MSC, PhD,

Correspondence: Anthony P. Morrison, School of Psychological Sciences,

University of Manchester, Oxford Road, Manchester M13 9PL, UK. Email:

tony.morrison@manchester.ac.uk

First received 20 Nov 2012, final revision 1 Mar 2013, accepted 17 Apr 2013

\section{Funding}

This research was supported by funding from the Medical Research Council (G0500264) and the Department of Health.

\section{Acknowledgements}

We thank the Mental Health Research Network, the Scottish Mental Health Research Network, and the OpenCDMS team for their support and assistance. We also wish to thank the other EDIE-2 investigators (Richard Bentall, Graham Dunn, Linda Davies, and Shon Lewis) and our trial therapists for their involvement in the trial and the EDIE-2 research assistants for their data collection work.

\section{References}

1 Yung AR, McGorry PD, McFarlane C, Jackson HJ, Patton GC, Rakkar A. Monitoring and care of young people at incipient risk of psychosis. Schizophr Bull 1996; 22: 283-303.

2 Yang LH, Wonpat-Borja AJ, Opler MG, Corcoran CM. Potential stigma associated with inclusion of the psychosis risk syndrome in the DSM-V: an empirical question. Schizophr Res 2010; 120: 42-8.

3 Corcoran CM, First MB, Cornblatt BA. The psychosis risk syndrome and its proposed inclusion in the DSM-V: a risk/benefit analysis. Schizophr Res 2010; 120: $16-22$

4 Morrison AP, Byrne RE, Bentall RP. DSM-5 and the 'Psychosis Risk Syndrome': whose best interests would it serve? Psychosis 2010; 2: 96-9.

5 Addington J, Epstein I, Liu L, French P, Boydell KM, Zipursky RB. A randomized controlled trial of cognitive behavioral therapy for individuals at clinical high risk of psychosis. Schizophr Res 2011; 125: 54-61.
6 McGlashan TH, Zipursky RB, Perkins D, Addington J, Miller T, Woods SW, et al. Randomized, double-blind trial of olanzapine versus placebo in patients prodromally symptomatic for psychosis. Am J Psychiatry 2006; 163: 790-9.

7 McGorry PD, Yung AR, Phillips L, Yuen HP, Francey S, Cosgrave EM, et al. Randomized controlled trial of interventions designed to reduce the risk of progression to first-episode psychosis in a clinical sample with subthreshold symptoms. Arch Gen Psychiatry 2002; 59: 921-8.

8 Morrison AP, French P, Walford L, Lewis SW, Kilcommons A, Green J, et al. Cognitive therapy for the prevention of psychosis in people at ultra-high risk. Randomised controlled trial. Br J Psychiatry 2004; 185: 291-7.

9 Amminger GP, Schafer MR, Papageorgiou K, Klier CM, Cotton SM, Harrigan $\mathrm{SM}$, et al. Long-chain omega-3 fatty acids for indicated prevention of psychotic disorders: a randomized, placebo-controlled trial. Arch Gen Psychiatry 2010; 67: 146-54.

10 Bechdolf A, Wagner M, Ruhrmann S, Harrigan S, Putzfeld V, Pukrop R, et al. Preventing progression to first-episode psychosis in early initial prodromal states. Br J Psychiatry 2012; 200: 22-9.

11 Yung AR, Phillips $\amalg$, Nelson B, Francey SM, PanYuen H, Simmons MB, et al. Randomized controlled trial of interventions for young people at ultra high risk for psychosis: 6-month analysis. J Clin Psychiatry 2011; 72: 430-40.

12 Morrison AP, French P, Stewart S, Birchwood M, Fowler B, Gumley Al. Early Detection and Intervention Evaluation for people at risk of psychosis (EDIE-2): a multisite randomised controlled trial of cognitive therapy for at risk mental states. BMJ 2012; 344: e2233.

13 Stafford MR, Jackson H, Mayo-Wilson E, Morrison AP, Kendall T. Early interventions to prevent psychosis: systematic review and meta-analysis. BMJ 2013; 346: f185.

14 Warner R. The prevention of schizophrenia: what interventions are safe and effective? Schizophr Bull 2001; 27: 551-62.

15 Corcoran C, Malaspina D, Hercher L. Prodromal interventions for schizophrenia vulnerability: the risks of being "at risk". Schizophr Res 2005; 73: 173-84.

16 French P, Morrison AP. Early Detection and Cognitive Therapy for People at High Risk of Developing Psychosis: A Treatment Approach. Wiley, 2004.

17 Bentall RP, Morrison AP. More harm than good: the case against using antipsychotic drugs to prevent severe mental illness. J Ment Health 2002; 11: 351-65.

18 Link BG, Yang LH, Phelan JC, Collins PY. Measuring mental illness stigma. Schizophr Bull 2004; 30: 511-41.

19 Corrigan PW, Watson AC. The paradox of self-stigma and mental illness. Clin Psychol 2002; 9: 35-53.

20 Ritsher JB, Otilingam PG, Grajales M. Internalized stigma of mental illness: psychometric properties of a new measure. Psychiatry Res 2003; 121: $31-49$.

21 Yung AR, Yuen HP, McGorry PD, Phillips LJ, Kelly D, Dell'Olio M, et al Mapping the onset of psychosis - the comprehensive assessment of at risk mental states (CAARMS). Aust N Z J Psychiatry 2005; 39: 964-71.

22 Birchwood M, Mason R, MacMillan F, Healy J. Depression, demoralization and control over psychotic illness: a comparison of depressed and nondepressed patients with a chronic psychosis. Psychol Med 1993; 23: $387-95$

23 Birchwood M, Jackson C, Brunet K, Holden J, Barton K. Personal Beliefs about Illness Questionnaire-Revised (PBIQ-R): reliability and validation in a first episode sample. Br J Clin Psychology 2012; 51: 448-58.

24 Morrison AP. The interpretation of intrusions in psychosis: an integrative cognitive approach to hallucinations and delusions. Behav Cogn Psychother 2001; 29: 257-76.

25 Little RJA, Rubin DB. Statistical Analysis with Missing Data. John Wiley and Sons, 2002.

26 Maracy M, Dunn G. Estimating dose-response effects in psychological treatment trials: the role of instrumental variables. Stat Methods Med Res 2011; 20: 191-215.

27 Morrison AP, Stewart S, French P, Bentall RP, Birchwood M, Byrne R, et al. Early Detection and Intervention Evaluation for people at high-risk of psychosis-2 (EDIE-2): trial rationale, design and baseline characteristics. Early Interv Psychiatry 2011; 5: 24-32.

28 Carpenter WT. Anticipating DSM-V: should psychosis risk become a diagnostic class? Schizophr Bull 2009; 35: 841-3.

29 Thornicroft G, Brohan E, Rose D, Sartorius N, Leese M. Global pattern of experienced and anticipated discrimination against people with schizophrenia: a cross-sectional survey. Lancet 2009; 373: 408-15.

30 Goffman E. Stigma: Notes on the Management of Spoiled Identity. Penguin Books, 1963. 
31 Brohan E, Elgie R, Sartorius N, Thornicroft G. Self-stigma, empowerment and perceived discrimination among people with schizophrenia in 14 European countries: the GAMIAN-Europe study. Schizophr Res 2010; 122: 232-8.

32 Thornicroft G. Most people with mental illness are not treated. Lancet 2007 370: $807-8$.

33 Thornicroft G, Rose D, Kassam A. Discrimination in health care against people with mental illness. Int Rev Psychiatry 2007; 19: 113-22.

34 Corrigan PW, Watson AC, Barr L. The self-stigma of mental illness: implications for self-esteem and self-efficacy. J Soc Clin Psychol 2006; 25: 875-84
35 Birchwood M, Trower P, Brunet K, Gilbert P, Iqbal Z, Jackson C. Social anxiety and the shame of psychosis: a study in first episode psychosis. Behav Res Ther 2007; 45: 1025-37.

36 Livingston JD, Boyd JE. Correlates and consequences of internalized stigma for people living with mental illness: a systematic review and meta-analysis. Soc Sci Med 2010; 71: 2150-61.

37 Byrne R, Morrison AP. Young people at risk of psychosis: their subjective experiences of monitoring and cognitive behaviour therapy in the EDIE 2 trial. Psychol Psychother, in press.

38 International Early Psychosis Association Writing Group. International clinical practice guidelines for early psychosis. Br J Psychiatry 2005; 187 (suppl 48): s120-4.

\section{poems \\ by doctors}

... and thus it came to pass...

Inanimate mechanical forces

Rampage through the central nervous systems

of once beautiful people

Distorting affects and salience

To such an extent

That intentional self-harm by exposure to organic solvents, hot vapours, wood preservatives

Or firearm discharge

Take place

Whether in private homes

Public administrative areas

Or institutional places of residence 